\title{
Nocardia asteroides keratitis A case report
}

\author{
R. A. RALPH, M. A. LEMP, AND G. LISS \\ From the Cornea Service, Department of Ophthalmology, \\ Georgetown University Medical Center, Washington, DC
}

Problems in management of ulcerative keratitis may arise from delays in diagnosis and from inadequate response to treatment. In the present case, a corneal injury was followed by Nocardia asteroides keratitis. The organism isolated was sensitive in vitro to both sulphonamides and penicillin, but there was no satisfactory clinical improvement with sulphonamides alone. Good response to treatment was delayed until topical penicillin $\mathrm{G}$ was added to the regimen.

\section{Case report}

An I I-year-old girl on vacation sustained an abrasion of the right eye from a fishline sinker on 26 June 1974. The eye was patched over a neomycin-polymyxin Bbacitracin ointment in the emergency ward of a local hospital. Because of persistent pain, she was examined on 2 July by her own ophthalmologist, who noted aqueous flare and cells, as well as many fine, cellular keratitic precipitates. The corneal surface centrally had a smooth, gentle concavity, without infiltrate or staining with fluorescein. To the topical antibiotics were added homatropine 5 per cent and prednisolone I per cent solutions, each four times daily, for treatment of the iritis. Diagnostic scraping for bacterial and fungal culture was performed on 15 July because of the reappearance of an epithelial defect. When seen in corneal consultation on 19 July the patient was using gentamicin 0.3 per cent solution every 2 hours, together with the homatropine 5 per cent and prednisolone I per cent solutions, each four times daily.

Uncorrected visual acuity in the right eye was $6 / 15$, while that in the left was $6 / 6$. The previously-noted epithelial defect had closed, but numerous greyishwhite subepithelial or superficial stromal opacities were concentrated in a central, ring-like distribution of about $5 \mathrm{~mm}$ in diameter (Fig. I). After repeating the diagnostic scrapings, gentamicin drops were reduced to four times daily, prednisolone I per cent to twice daily, and erythromycin ointment four times a day was added.

Three days later, on 22 July, cultures from the original scraping demonstrated growth of an acid-fast

Address for reprints : Robert A. Ralph, MD Department of Ophthalmology, Georgetown University Medical Center, 3800 Reservoir Road, N.W., Washington, DC 20007 USA organism with long, branching, fragmenting hyphae. Colonies were at first chalky white, later becoming orange, and they grew on both the bottom and the surface of Sabouraud's broth. At room temperature, the organism grew slowly on Sabouraud's agar without antibiotics, on a blood agar plate (10 per cent sheep blood), and on Löwenstein-Jensen medium. It failed to grow on the Sabouraud's plate in the presence of inhibitory antibiotics. Growth on all of the above media, including those containing antibiotics, was rapid at a temperature of $35^{\circ} \mathrm{C}$. There was no growth on any medium at $45^{\circ} \mathrm{C}$. The organism was positive for nitrate, catalase, and urea. It failed to hydrolyze casein, xanthine, trypsin, and starch. Guinea-pig intraperitoneal inoculation allowed recovery of acid-fast organisms in 7 to 8 days. Because of the above cultural and biochemical patterns, the organism was classified as $N$. asteroides, sensitive to penicillin, ampicillin, streptomycin, and triple sulpha.

The eye was more congested by 22 July and there was generalized corneal oedema, consistent with a visual acuity of perception of light with projection. Scrapings repeated at that time demonstrated long, branching hyphae (Fig. 2), and eventually grew out $N$. asteroides. Subconjunctival ampicillin $125 \mathrm{mg}$ was injected, followed by 30 per cent sodium sulphacetamide drops. every hour and 1o per cent sodium sulphacetamide. ointment at bedtime.

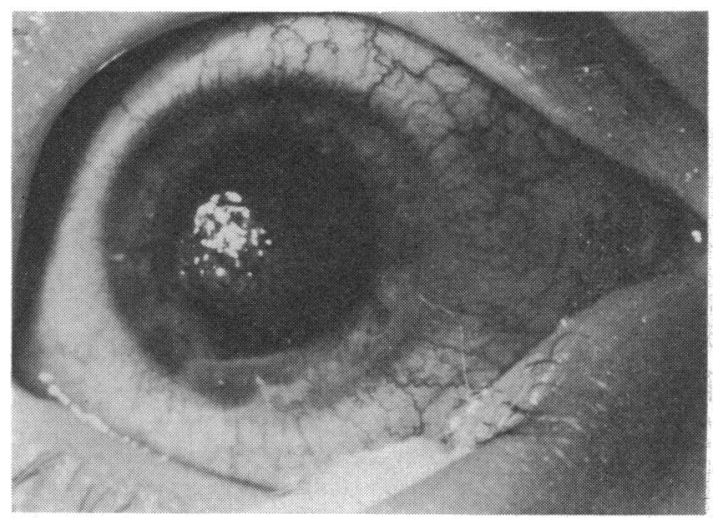

FIG. I Nocardia asteroides keratitis. Three weeks after injury with a fishline sinker. Multiple superficial stromal opacities in ring-like distribution 


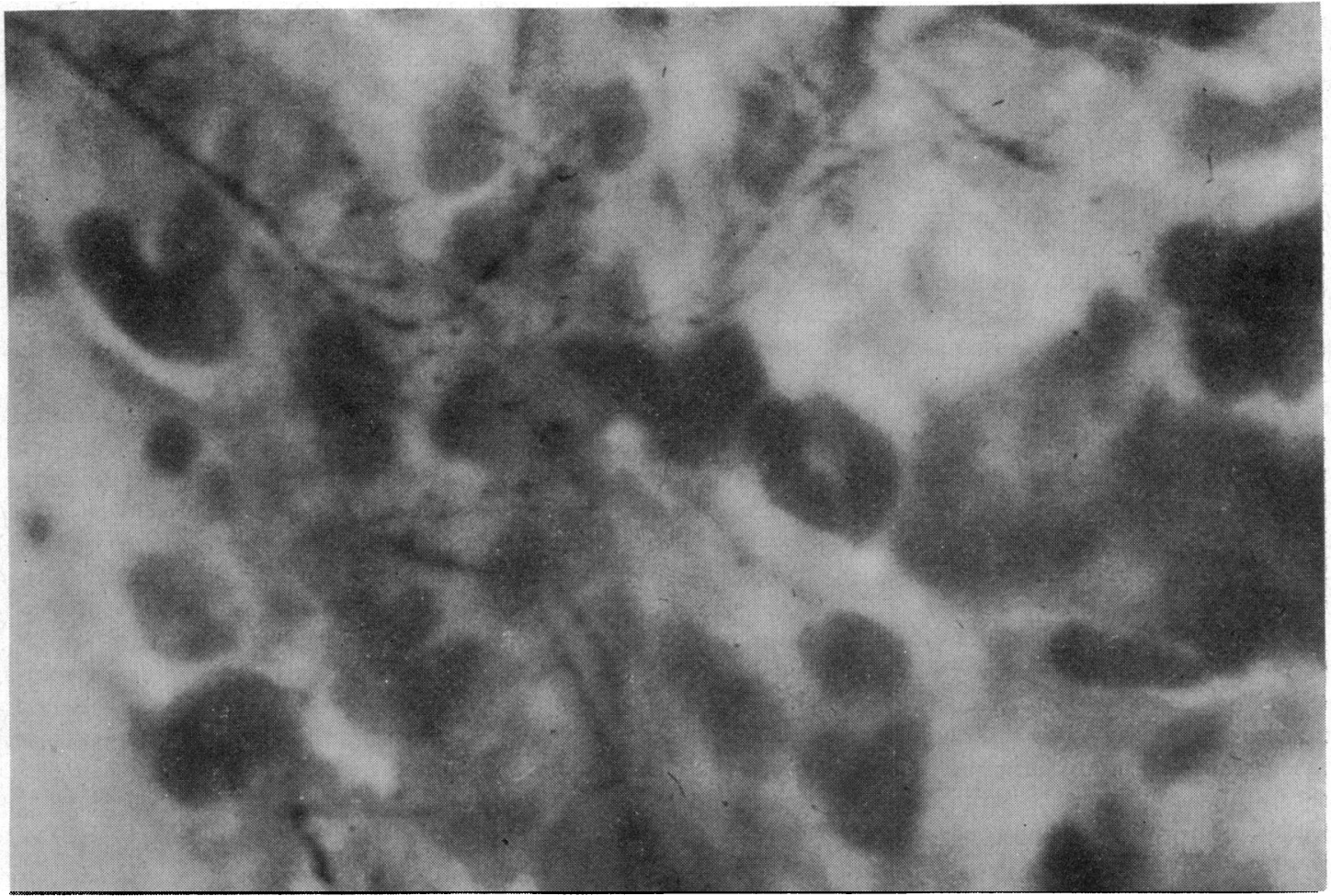

FIG. 2 Nocardia asteroides keratitis. Scraping of lesion reveals branching hyphae among inflammatory cells.

Gram stain. $\times 200$

The cornea improved rapidly and 5 days later there remained only a persistent ring of subepithelial punctate opacities. The lesion was once again scraped, and penicillin $\mathrm{G}$ drops 294000 units per $\mathrm{ml}$ (in an artificial tear solution) were added, alternating hourly with the sodium sulphacetamide drops.

The remaining opacities cleared gradually, and the epithelium healed. When examined on 12 August 1974, the uncorrected visual acuity was $6 / 12$; there was a central superficial stromal scar, with an overlying epithelium (Fig. 3). Topical penicillin was discontinued, and the sodium sulphacetamide was gradually stopped during the next 2 weeks.

\section{Discussion}

Few case reports have been published describing $N$. asteroides keratitis (Schardt, Unsworth, and Hayes, 1956; Newmark, Polack, and Ellison, I97 I). In each instance, however, injury occurring outdoors has been a common factor, since a frequent source of Nocardia is the soil. Previous reports have not emphasized the unusual appearance of the involved eye. Lesions affect only the superficial onethird of the corneal stroma. Adjacent opacities may be arranged in a confluent, ring-like distribution, as in the present case. Eventual resolution leaves superficial stromal scarring and surface irregularities.
Nocardia asteroides is composed of gram positive, weakly acid-fast filamentous forms, with diameters of 0.5 to $1 \cdot 0 \mu \mathrm{m}$ (Fig. 2). In early cultures, branching of filaments at right-angles is distinctive, but in older colonies these tend to fragment, producing bacillary and coccoidal forms, more closely resembling bacteria than fungi (Meyer, Font, and Shaver,

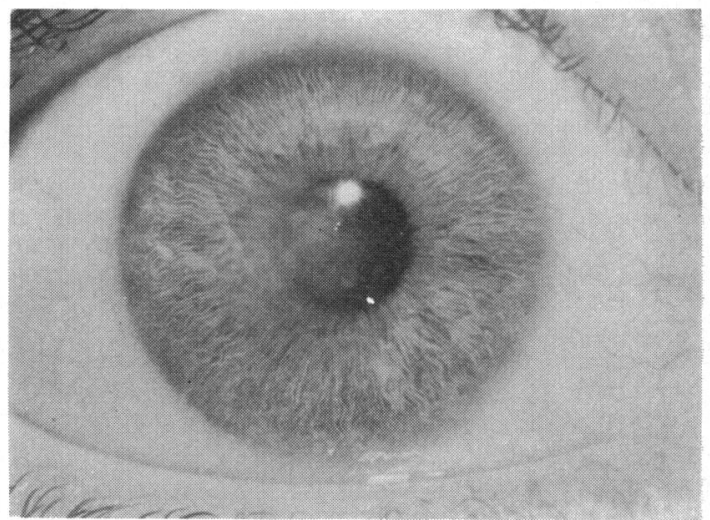

FIG. 3 Nocardia asteroides keratitis. After therapy with topical penicillin $G$. The epithelium has grown over persistent superficial stromal opacities 
1970). The acid-fast characteristics are more obvious in tissue, and less evident in culture. Blood and Sabouraud's agar are good substrates for pathogenic Nocardia, which generally mature satisfactorily at temperatures between $35^{\circ}$ and $37^{\circ} \mathrm{C}$. Because they are slow growing, however, colonies may not be evident on the culture plates for several days. Culture media containing chloramphenicol, penicillin, and streptomycin are inhibitory to Nocardia, and should not be used for primary isolation if this organism is suspected (Murray, Finegold, Froman, and Will, 1961).

In most cases, Nocardia is sensitive to penicillin, sulphonamides, and tetracycline. The case reported by Schardt and others (1956) responded to systemic and topical oxytetracycline, while that of Newmark and others (I97I) was treated successfully with systemic sulphadiazine, together with topical sulphacetamide and tetracycline.

In the present case, contamination of an injured cornea with $N$. asteroides had led to a symptomatic infection by the fifth day after the accident. As in the patient described by Newmark and others (1971), persistence of inflammation and worsening on a combination of topical antibiotics and corticosteroids prompted a vigorous search for an atypical pathogen. Indeed, the offending organism was identified, but only after the delay of one week that is often characteristic of the slow-growing
Nocardia. Although sensitivity studies suggested that subconjunctival ampicillin and hourly sodium sulphacetamide would constitute effective treatment, only when penicillin $G$ drops were added did the cornea clear maximally and the epithelium heal.

\section{Summary}

An I I-year-old girl developed a corneal ulcer five days after sustaining a corneal abrasion from a fishline sinker. After more than three weeks, the epithelial defect had closed, but superficial stromal opacities in a ring-like distribution had not cleared on topical gentamicin, prednisolone I per cent, and atropine. Several scrapings disclosed the presence of branching hyphae on smear, eventually identified as Nocardia asteroides by culture. Although sensitivity studies suggested that subconjunctival ampicillin and hourly sodium sulphacetamide would constitute effective treatment, resolution was only partial on this regimen. Only when penicillin $\mathrm{G}$ drops were added did the cornea clear maximally and the epithelium heal.

The authors wish to express their thanks to Joseph C Stone, Mycologist, Wheaton, Maryland, USA, for his painstaking efforts in conclusively identifying the offending organism.

\section{References}

MEYeR, S. L., FONT, R. L., and SHAVER, R. R. (1970) Arch. Ophthal., 83, 536

MURRAY, J. F., FINEgold, s. M., FROMAN, s., and will, D. w. (I96r) Amer. Rev. resp. Dis., 83, 3 I 5

newmark, e., Polack, f. M., and ellison, A. c. (1971) Amer. F. Ophthal., 72, 81 3

SCHARDT, W. M., UNSWORTH, A. C., and HAYES, C. v. (1956) Ibid., 42, 303 